\title{
Nuevo registro de Hypopachus variolosus (Cope, 1866) (Anura: Microhylidae) en el departamento de La Libertad, El Salvador
}

\author{
New record of Hypopachus variolosus (Cope, 1866) \\ (Anura: Microhylidae) in the department La Libertad, EI Salvador
}

\author{
Néstor Esaú Ruballo-Marroquín', Emanuel Stanley Morán-Hidalgo²
}

\begin{abstract}
Resumen
En esta nota se presenta la ampliación de la distribución geográfica y altitudinal de una especie de Microhylidae, amenazada en El Salvador, Hypopachus variolosus. Por medio de la búsqueda activa por encuentro visual, en los diferentes hábitat de la planicie costera de la Cordillera del Bálsamo, departamento de La Libertad, se registró un individuo de Hypopachus variolosus, a 28 m de elevación, ocupando los microhábitat entre la hojarasca. Este hallazgo es importante, se ubica una nueva población de esta especie amenazada por el crecimiento de la frontera agrícola y ganadera en EI Salvador

Palabras clave: Cordillera del Bálsamo, Microhylidae, Planicie costera.

Abstract

This note presents the extension of the geographical and altitudinal distribution of a species of microhylid, Threatened in El Salvador, Hypopachus variolosus. Through the active search and visual encounter in different habitats in the coastal plain of the Cordillera del Balsamo, Department of La Libertad, was an individual of Hypopachus variolosus, 28 m elevation, occupying between litter microhabitat. This discovery is important, is located a new population of this threatened species by the growth of the agricultural frontier in El Salvador.
\end{abstract}

Keywords: Coastal plain, Microhylidae, Mountains the Balsamo.

\section{Introducción}

Hypopachus variolosus es una especie cuyas poblaciones se encuentran distribuidas en la vertiente del Atlántico, desde el sur de Texas (EE.UU), hasta Costa Rica, y en la vertiente del Pacífico, desde el sur de Sonora (México), hasta Costa Rica, en un gradiente altitudinal entre el nivel del mar y los 1610 $\mathrm{m}$ de altitud (Köhler 2011). En El Salvador está distribuida entre los 700 y 1240 m de altitud (Köhler et al. 2006).

Según recolectas históricas realizadas en El Salvador, resguardadas en los museos Museo de Historia Natural de El Salvador (MUHNES), Kansas University (KU), Seckemberg Museum of Franckfurt (SMF) y University of Utah Natural History Museum
(UU). H. variolosus se distribuye en el departamento de San Miguel a 740 msnm, Volcán de San Miguel (KU 291269-72; 291387); MUHNES (C-301436-39). San Salvador: Instituto Tropical de Investigaciones Científicas (KU 62004-23; UU 4908). San Vicente: a $1240 \mathrm{msnm}$, Finca El Carmen $\left(13^{\circ} 36.8^{\prime} \mathrm{N}\right.$, -88 50.3’W; SMF 87370). Usulután: a $1100 \mathrm{msnm}$ Cerro El Tigre $\left(13^{\circ} 28.37^{\prime} \mathrm{N}, 88^{\circ} 26.21^{\prime} \mathrm{W}\right.$; KU 289842), (Köhler et al. 2006).

El Área Natural Protegida Parque Walter Thilo Deininger, está ubicada en el municipio de La Libertad, departamento de La Libertad, formando parte de zona de conservación Costa del Bálsamo (MARN 2011, Figura1). En la zona se desarrollan los ecosistemas de bosque tropical semideciduo latifoliado de tierras bajas, bien drenado, secunda-

Colonia Bosques de Prusia Pje. El Roble, San Salvador, El Salvador. e-mail: ruballonestor@yahoo.com

Urbanización Prados de Venecia I, San Salvador, El Salvador. e-mail: stanley hidalgo@yahoo.com

Fecha recepción: Febrero 19, 2013

Fecha aprobación: Abril 23, 2013

(c) Rev. Biodivers. Neotrop. 2013; 3 (1): 65-8 


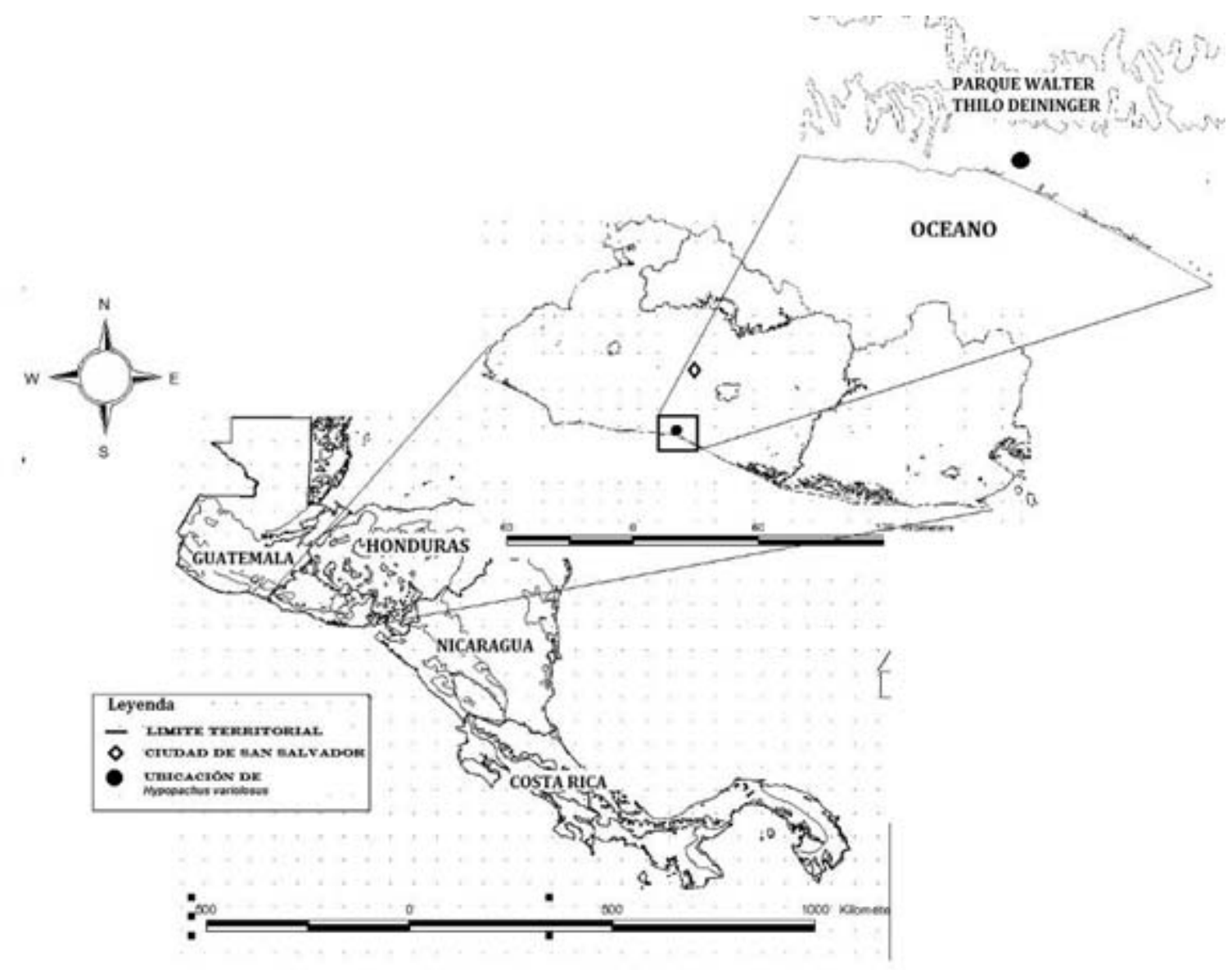

Figura 1. Ubicación de la nueva localidad de Hypopachus variolosus para El Salvador, en el Área Natural Protegida Parque Walter Thilo Deininger, municipio de La Libertad, departamento de La Libertad, agosto del 2012.

rio $\mathrm{y} / \mathrm{o}$ intervenido y bosque tropical deciduo latifoliado de tierras bajas, bien drenado, secundario y/o intervenido, manteniendo formaciones de bosque deciduo, bosque siempreverde latifoliado y bosque ripario a la largo de los ríos que atraviesan el área (Programa REDD CCAD GIZ 2011).

El muestreo se llevó a cabo el 31 de agosto de 2012, considerada la temporada lluviosa para la región, y realizado a través de la búsqueda intensiva y encuentro visual, en el marco del primer inventario de anfibios del Parque Walter Thilo Deininger. Se registró un individuo adulto de Hypopachus variolosus, aproximadamente a las 21:30 horas, en el sendero «Cueva del Encanto», que bordea una de las zonas de cultivo, adyacentes al parque $\left(13.48578^{\circ}\right.$ $\mathrm{N},-89.26933^{\circ} \mathrm{W} ; 28 \mathrm{~m}$ de elevación. El individuo fue registrado en un área cubierta por vegetación semidecidua intervenida y zona de cultivo, con predominante suelo arenoso.
El ejemplar encontrado (Figura 2) fue fotografiado e identificado en el sitio con el apoyo de las claves taxonómicas disponibles en la guías de campo (Köhler et al. 2006; McCraine y Castañeda 2007).

El parque Deininger es un área natural de gran importancia al incluirse entre los bosques deciduos, ya que, estos bosques pueden albergar una alta diversidad de especies en El Salvador (Herrera 2005), se reconoce que existen más de 34,000 ha de bosque secos (deciduos), pero sólo 9,700 ha poseen algún nivel de protección como áreas naturales protegidas (citados en Herrera et al. 2006); de esta cifra, 2,521 ha de Bosque Tropical Deciduo pertenecen al Área de Conservación Costa del Bálsamo.

Este nuevo registro, ayuda a contribuir sobre la distribución de los anfibios en El Salvador, al encontrarse esta especie en estado de «amenazada» en el país (MARN 2009), por la destrucción de su hábitat natural y el poco conocimiento científico de esta es- 


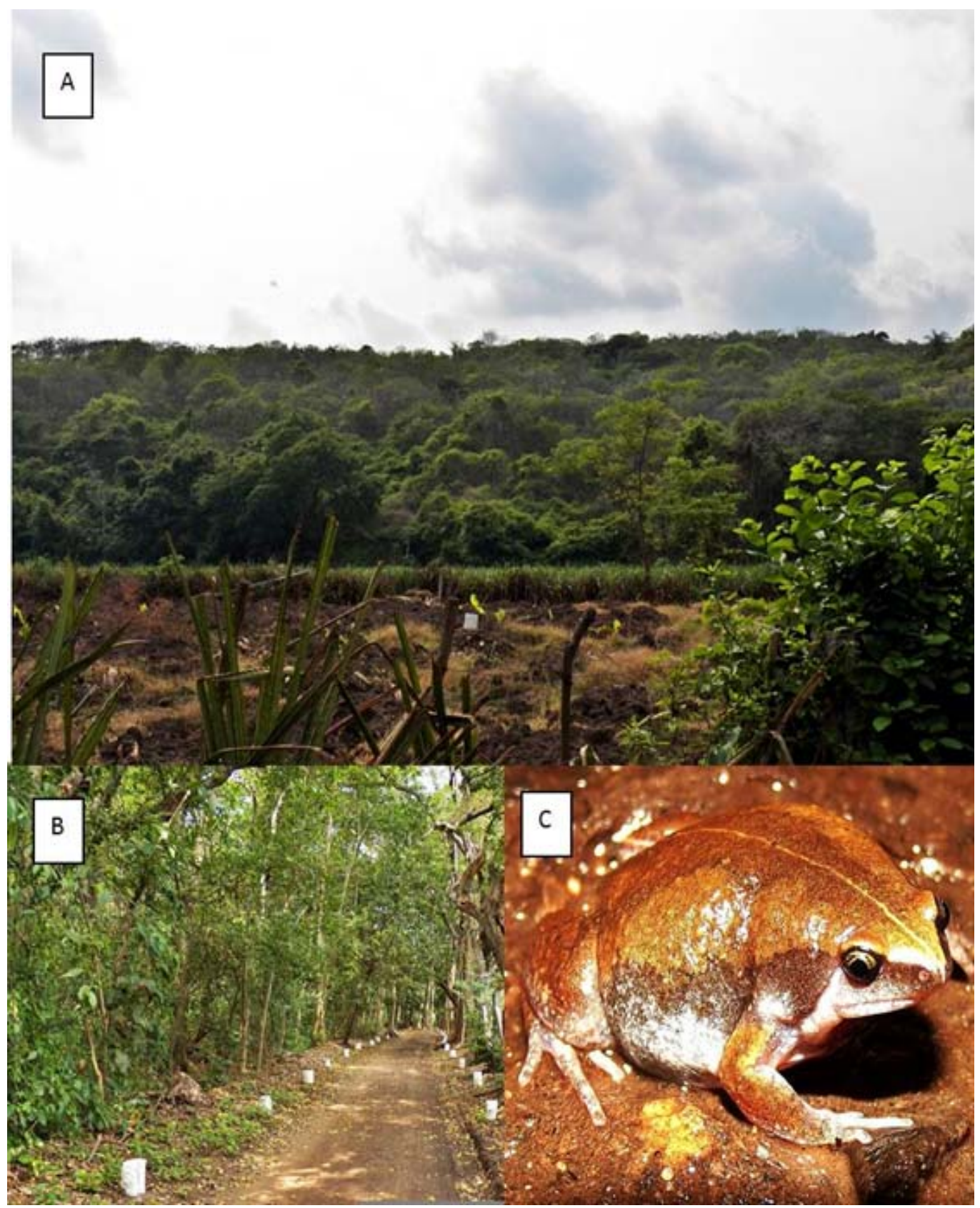

Figura 2. Ejemplar de Hypopachus variolosus registrado en el Área Natural Protegida Parque Walter Thilo Deininger, municipio de La Libertad, departamento de La Libertad, El Salvador, agosto del 2012. A. Panorámica del Área Natural Protegida Parque Walter Thilo Deininger, B. Sitio del avistamiento sendero «Cueva del Encanto», C. Individuo adulto de Hypopachus variolosus.

pecie. Según Santos-Barrera et al. (2010) se encuentra en la categoría de menor preocupación (LC) a nivel mundial.

Con este hallazgo también se amplía el rango de distribución altitudinal hacia la zona de planicie costera porque para El Salvador, se conocía la distribu- ción de $H$. variolosus entre 700-1240 m de elevación (Köhler et al. 2006).

Es importante este registro, porque el parque Deininger se encuentra rodeado de sistema agrícola y según (Young et al. 2001), las principales amenazas para las especies de anfibios son la transforma- 
ción y la pérdida de los hábitats, aunque otras causas como enfermedades o contaminación (uso de fertilizantes y pesticidas), también tienen un impacto importante.

Conocer el potencial faunístico de las Áreas Naturales Protegidas, es de suma importancia, si se tiene en cuenta que existen datos que indican que más de 800 especies de anfibios tienen su distribución por fuera de las áreas naturales protegidas que existen actualmente a nivel global (Rodrígues et al. 2004). El Salvador no escapa a esta realidad ya que son pocos los estudios, en los complejos protegidos y esto ha ocasionado que gran parte de su riqueza faunística, entre ellos la especie Hypopachus variolosus, se halla recolectado y/o registrado solo por fuera de estas áreas en el país (Köhler et al. 2006).

\section{Agradecimientos}

Al Licenciado Antonio Escobar Macal, Jefe Unidad Centros Recreativos, del Instituto de Turismo de El Salvador (ISTU), por el permiso y para ingresar al área natural protegida para realizar está investigación, a Jacqueline Rosales, estudiante de Escuela de Biología, Universidad de El Salvador por el apoyo en la fase de campo.

\section{Literatura citada}

Herrera N, Henríquez V, Rivera A. M. 2005. Contribuciones al conocimiento de la herpetofauna de El Salvador. Mesoamericana. Bol Soc Mesoame Biol Conserv. 9 (3): $1-6$.

Köhler G. 2011. Amphibians of Central America. Offenbach: Herpeton Verlag. 376 pp.

Köhler G, Veselý M, Greenbaun E. 2006. The amphibians and reptiles of El Salvador. Malabar: Krieger Publ Co. 238 pp.

McCraine JR, Castañeda EF. 2007. Guía de campo de anfibios de Honduras. Salt Lake City: Bibliomanía. 304 pp.

Ministerio de Medio Ambiente y Recursos Naturales (MARN) 2009. Listado oficial de especies de vida silvestre, amenazadas o en peligro de extinción. Acuerdo $N^{\circ} 36$, Diario Oficial $N^{\circ} 102$, Tomo $\mathrm{N}^{\circ} 383$.

Ministerio de Medio Ambiente y Recursos Naturales (MARN). 2011. Estudio de racionalización y priorización del sistema de áreas naturales protegidas de la República de El Salvador. San Salvador: World Institute for Conservation and Environment. 254 pp.

Programa REDD-CCAD-GIZ. 2011. Tipos de bosque y contexto del mapeo de la cobertura forestal en Centro América y República Dominicana. Antigua: Deutsche Gesellsachaft für Internationale Zusammenarbeit. 18 pp.

Rodrígues ASL, Andelman SJ, Bakarr MI, et al. 2004. Effectiveness of global protected area network in representing species diversity. Nature. 428: 640-3.

Santos-Barrera G, Hammerson G, Chaves G, et al. 2010. Hypopachus variolosus. [en línea]. IUCN Red List of Threatened Species. (Consultado 26 de abril 2013). URL disponible en: http://www.iucnredlist.org

Young BR, Lips KR, Reaser JK, et al. 2001. Population declines and priorities for amphibian conservation in Latin America. Consevat Biol 15: 1213-23. 